\title{
ON SOME LOCAL GEOMETRY OF MUSIELAK-ORLICZ SEQUENCE SPACES EQUIPPED WITH THE LUXEMBURG NORM
}

\author{
YUNAN CUI \\ ChongQing University of Posts and Telecommunications \\ ChongQing 400065, P.R. China \\ E-mail: cuiya@hrbust.edu.cn \\ YANHONG LI \\ Beijing University of Technology, Beijing 100080, P.R. China \\ MINGXIA ZOU \\ Department of Mathematics, Harbin University of Science and Technology \\ Harbin 150080, P.R. China
}

\begin{abstract}
Criteria for strong U-points, compactly locally uniformly rotund points, weakly compactly locally uniformly rotund points and locally uniformly rotund points in Musielak-Orlicz sequence spaces equipped with the Luxemburg norm are given.
\end{abstract}

1. Introduction. Throughout this paper, $X$ denotes a Banach space and $X^{*}$ denotes its dual space. By $B(X)$ and $S(X)$ we denote the closed unit ball and the unit sphere of $X$, respectively.

Definition 1. A point $x \in S(X)$ is said to be an extreme point if for every $y, z \in S(X)$ with $x=\frac{y+z}{2}$, we have $y=z=x$.

A Banach space $X$ is said to be rotund $(X \in(R)$ for short) if every point on $S(X)$ is an extreme point.

Definition 2. A point $x \in S(X)$ is said to be a strong U-point (SU-point for short) if for any $y \in S(X)$ with $\|y+x\|=2$ we have $x=y$.

2000 Mathematics Subject Classification: 46B20, 46E30.

Key words and phrases: Musielak-Orlicz sequence spaces, SU-point, CLUR-point, WCLURpoint, LUR-point.

Supported by NSF of CHINA.

The paper is in final form and no version of it will be published elsewhere. 
It is obvious that a Banach space $X$ is rotund if and only if every $x \in S(X)$ is a SU-point.

Definition 3. A point $x \in S(X)$ is said to be a locally uniformly rotund point (LURpoint for short) if for any sequence $\left\{x_{n}\right\}_{n=1}^{\infty}$ in $S(X)$ with $\lim _{n \rightarrow \infty}\left\|x_{n}+x\right\|=2$, we have $\lim _{n \rightarrow \infty}\left\|x_{n}-x\right\|=0$.

DeFINITION 4. A point $x \in S(X)$ is said to be a weakly compactly locally uniformly rotund point (WCLUR-point for short) if for any sequence $\left\{x_{n}\right\}_{n=1}^{\infty}$ in $S(X)$ with $\lim _{n \rightarrow \infty}\left\|x_{n}+x\right\|$ $=2$, there exist an $x^{\prime} \in S(X)$ and a subsequence $\left\{x_{n}^{\prime}\right\}$ of $\left\{x_{n}\right\}$ such that $x_{n}^{\prime}$ convergent to $x^{\prime}$ weakly $\left(x_{n}^{\prime} \rightarrow^{w} x^{\prime}\right.$ for short $)$.

Definition 5. A point $x \in S(X)$ is said to be a compactly locally uniform rotund point (CLUR-point for short) if for any sequence $\left\{x_{n}\right\}_{n=1}^{\infty}$ in $S(X)$ with $\lim _{n \rightarrow \infty}\left\|x_{n}+x\right\|=2$, the sequence $\left\{x_{n}\right\}$ is compact in $B(X)$.

Definition 6. A Banach space $X$ is said to have H-property if the weak convergence and the convergence in norm coincide in $S(X)$.

For these geometric notions and their role in mathematics we refer to the monographs [1] and [2].

The function sequence $M=\left(M_{i}\right)_{i=1}^{\infty}$ is called a Musielak-Orlicz function provided that for any $i \in \mathbb{N}, M_{i}:(-\infty,+\infty) \rightarrow[0,+\infty)$ is even, convex, left continuous on $[0,+\infty), M_{i}(0)=0$ and there exists $u_{i}>0$ such that $M_{i}\left(u_{i}\right)<\infty$. By $N=\left(N_{i}\right)_{i=1}^{\infty}$ we denote the Musielak-Orlicz function conjugate to $M=\left(M_{i}\right)$ in the sense of Young, i.e.

$$
N_{i}(u)=\sup _{v>0}\left\{|u| v-M_{i}(v)\right\}
$$

for each $u \in \mathbb{R}$ and $i \in \mathbb{N}$. Furthermore, $P=\left(p_{i}\right)$ is the right derivative of $M=\left(M_{i}\right)$, i.e. $p_{i}$ is the right derivative of $M_{i}$ for every $i \in \mathbb{N}$.

By $l^{0}$ we denote the space of all sequences $x=(x(i))_{i=1}^{\infty}$ of reals. For a given MusielakOrlicz function $M=\left(M_{i}\right)$ we define the Musielak-Orlicz sequence space $l_{M}$ by

$$
l_{M}=\left\{x \in l^{0}: \rho_{M}(\lambda x)<\infty \text { for some } \lambda>0\right\},
$$

where

$$
\rho_{M}(x)=\sum_{i=1}^{\infty} M_{i}(x(i)) \text { for any } x=(x(i)) \in l^{0} .
$$

This space equipped with the Luxemburg norm

$$
\|x\|=\inf \left\{\lambda>0: \rho_{M}(x / \lambda) \leq 1\right\}
$$

or with the Orlicz norm

$$
\|x\|^{0}=\sup \left\{\sum_{i} x(i) y(i): \rho_{N}(y) \leq 1\right\}=\inf _{k>0} \frac{1}{k}\left(1+\rho_{M}(k x)\right)
$$

is a Banach space (see [3]).

By $h_{M}$ we denote the subspace of $l_{M}$ defined by

$$
h_{M}=\left\{x \in l_{M}: \forall \lambda>0, \exists i_{0} \text { such that } \sum_{i>i_{0}} M_{i}(\lambda x(i))<\infty\right\} \text {. }
$$


To simplify notations, we put $l_{M}=\left(l_{M},\|\cdot\|\right)$ and $l_{M}^{0}=\left(l_{M},\|\cdot\|^{0}\right)$.

We say that the Musielak-Orlicz function $M=\left(M_{i}\right)$ satisfies the $\delta_{2}$-condition $\left(M \in \delta_{2}\right.$ for short) if there exist $a>0, k>0, i_{0} \in \mathbb{N}$ and a sequence $\left(c_{i}\right)_{i=i_{0}+1}^{\infty}$ in $[0,+\infty)$ with $\sum_{i>i_{0}}^{\infty} c_{i}<\infty$ such that

$$
M_{i}(2 u) \leq k M_{i}(u)+c_{i}
$$

for every $i \in \mathbb{N}$ and $u \in \mathbb{R}$ satisfying $M_{i}(u) \leq a$ (see [3]).

We say that the Musielak-Orlicz function $M=\left(M_{i}\right)$ satisfies the $\bar{\delta}_{2}$-condition $\left(M \in \bar{\delta}_{2}\right.$ for short) if its complementary function $N=\left(N_{i}\right)$ satisfies the $\delta_{2}$-condition.

For convenience, we introduce the following notions. For every $x \in l_{M}$ and $i \in \mathbb{N}$, we put

$$
\begin{aligned}
\xi(x) & =\inf \left\{\lambda>0: \text { there exists } i_{0} \text { such that } \sum_{i>i_{0}} M_{i}(x(i) / \lambda)<\infty\right\}, \\
e(i) & =\sup \left\{u \geq 0: M_{i}(u)=0\right\} \\
B(i) & =\sup \left\{u>0: M_{i}(u)<\infty\right\} .
\end{aligned}
$$

For every $i \in \mathbb{N}$, we say that a point $x \in \mathbb{R}$ is a strictly convex point of $M_{i}$ if $M_{i}\left(\frac{u+v}{2}\right)<\frac{1}{2}\left(M_{i}(u)+M_{i}(v)\right)$ whenever $x=\frac{u+v}{2}$ and $u \neq v$. We write then $x \in S C_{M_{i}}$. An interval $[a, b]^{(i)}$ is called a structurally affine interval for $M_{i}$ (or simply $S A I$ of $M_{i}$ ) provided that $M_{i}$ is affine on $[a, b]^{(i)}$ and it is not affine on $[a-\varepsilon, b]^{(i)}$ or $[a, b+\varepsilon]^{(i)}$ for any $\varepsilon>0$. Let $S A I\left(M_{i}\right)=\left\{\left[a_{n}, b_{n}\right]^{(i)}\right\}_{n=1}^{\infty}$. It is obvious that $S C_{M_{i}}=\mathbb{R} \backslash \cup_{n}\left[a_{n}, b_{n}\right]^{(i)}$, where $\left[a_{n}, b_{n}\right]^{(i)} \in S A I\left(M_{i}\right)$ for $n=1,2, \ldots$

For every $i \in \mathbb{N}$, denote

$$
\begin{aligned}
& S C_{M_{i}}^{-}=\left\{u \in S C_{M_{i}}: \exists \varepsilon>0 \text { such that } M_{i} \text { is affine on }[u, u+\varepsilon]\right\}, \\
& S C_{M_{i}}^{+}=\left\{u \in S C_{M_{i}}: \exists \varepsilon>0 \text { such that } M_{i} \text { is affine on }[u-\varepsilon, u]\right\}, \\
& S C_{M_{i}}^{0}=S C_{M_{i}} \backslash\left(S C_{M_{i}}^{+} \cup S C_{M_{i}}^{-}\right) .
\end{aligned}
$$

We first formulate several lemmas.

LEMMA $1([5]) .\left(h_{M}\right)^{*}=l_{N}^{0},\left(h_{M}^{0}\right)^{*}=l_{N}$.

LEMMA $2([5]) . h_{M}=l_{M}\left(\right.$ or $\left.h_{M}^{0}=l_{M}^{0}\right)$ if and only if $M \in \delta_{2}$.

LEMMA 3 ([4]). If $M \notin \bar{\delta}_{2}$, then there exist a sequence $0=m_{0}<m_{1}<m_{2}<\cdots$ and $u_{i}^{n}>0\left(i=m_{n-1}+1, \ldots, m_{n}\right)$ such that $M_{i}\left(u_{i}^{n}\right) \leq 1 / n$ and

$$
M_{i}\left(\frac{u_{i}^{n}}{2}\right)>\left(1-\frac{1}{n}\right) \frac{M_{i}\left(u_{i}^{n}\right)}{2}, \quad \sum_{i=m_{n-1}+1}^{m_{n}} M_{i}\left(u_{i}^{n}\right)>1, \quad n=1,2, \ldots
$$

LEMma 4 ([4]). $M \in \delta_{2}$ if and only if $\|x\|=1 \Leftrightarrow \rho_{M}(x)=1$.

Lemma 5. If $M \in \delta_{2},\|x\|=1,\left\|x_{n}\right\| \leq 1$ and $\left\|x_{n}+x\right\| \rightarrow 2(n \rightarrow \infty)$, then

$$
\lim _{n \rightarrow \infty} \rho_{M}\left(x_{n}\right)=\lim _{n \rightarrow \infty} \rho_{M}\left(\frac{x+x_{n}}{2}\right)=1 \text {. }
$$


Proof. We suppose that there exists $\varepsilon_{0}>0$ such that $\rho_{M}\left(x_{n}\right) \leq 1-\varepsilon_{0}(n=1,2, \ldots)$. Since $\frac{\left\|x_{n}+x\right\|}{2} \rightarrow 1$, for any $\eta>0$ there exists $n_{0} \in \mathbb{N}$ such that

$$
\left\|\frac{(1+\eta)\left(x+x_{n}\right)}{2}\right\|>1
$$

when $n \geq n_{0}$.

For any $\varepsilon>0$, by $M \in \delta_{2}$, there exist $\lambda_{0}>1, a>0$ and $c_{i}>0(i=1,2, \ldots)$ such that $\sum_{i=1}^{\infty} c_{i}<\infty$ and $M_{i}\left(\lambda_{0} u\right) \leq(1+\varepsilon) M_{i}(u)+c_{i}\left(\forall i \in \mathbb{N}, M_{i}(u) \leq a\right)$.

Take $i_{0} \in \mathbb{N}$ such that $\sum_{i>i_{0}} c_{i}<\varepsilon$ and $M_{i}(x(i)) \leq a \quad\left(i>i_{0}\right)$.

Take $\lambda_{0}^{\prime}>0$ with $1<\lambda_{0}^{\prime}<\lambda_{0}$ such that $\sum_{i=1}^{i_{0}}\left(M_{i}(\lambda x(i))-M_{i}(x(i))\right)<\varepsilon\left(1 \leq \lambda \leq \lambda_{0}^{\prime}\right)$. Therefore when $1 \leq \lambda \leq \lambda_{0}^{\prime}$, it follows that

$$
\begin{aligned}
\rho_{M}(\lambda x) & =\sum_{i=1}^{i_{0}} M_{i}(\lambda x(i))+\sum_{i>i_{0}} M_{i}(\lambda x(i)) \\
& \leq \sum_{i=1}^{i_{0}} M_{i}(\lambda x(i))+\varepsilon+\sum_{i>i_{0}}\left((1+\varepsilon) M_{i}(x(i))+c_{i}\right) \\
& \leq(1+\varepsilon) \rho_{M}(x)+2 \varepsilon
\end{aligned}
$$

i.e.

$$
\lim _{\lambda \rightarrow 1} \rho_{M}(\lambda x)=\rho_{M}(x) .
$$

Combining (1) with (2) we have

$$
\begin{aligned}
1 & <\rho_{M}\left(\frac{(1+\eta)\left(x+x_{n}\right)}{2}\right)=\rho_{M}\left(\frac{1+\eta}{2} x_{n}+\frac{1-\eta}{2} \frac{1+\eta}{1-\eta} x\right) \\
& \leq \frac{1+\eta}{2} \rho_{M}\left(x_{n}\right)+\frac{1-\eta}{2} \rho_{M}\left(\frac{1+\eta}{1-\eta} x\right) \\
& \leq \frac{1+\eta}{2}\left(1-\varepsilon_{0}\right)+\frac{1-\eta}{2}(1+o(\eta)) .
\end{aligned}
$$

Let $\eta \rightarrow 0$ to get $1 \leq \frac{1-\varepsilon_{0}}{2}+\frac{1}{2}$. This is a contradiction. So $\rho_{M}\left(x_{n}\right) \rightarrow 1(n \rightarrow \infty)$.

Using $\left\|\frac{x+\frac{x+x_{n}}{2}}{2}\right\|=\left\|\frac{3}{4} x+\frac{1}{4} x_{n}\right\| \rightarrow 1$, by the same argument as above we have $\rho_{M}\left(\frac{x+x_{n}}{2}\right) \rightarrow 1(n \rightarrow \infty)$.

Lemma 6. If $M \in \delta_{2}$ and $x_{n}(i) \rightarrow 0(i=1,2 \ldots)$, then $\left\|x_{n}\right\| \rightarrow 0 \Leftrightarrow \rho_{M}\left(x_{n}\right) \rightarrow 0$.

Proof. Since it is obvious that $\left\|x_{n}\right\| \rightarrow 0$ implies $\rho_{M}\left(x_{n}\right) \rightarrow 0$, we only need to prove that $\rho_{M}\left(x_{n}\right) \rightarrow 0$ implies $\left\|x_{n}\right\| \rightarrow 0(n \rightarrow \infty)$. For any $\varepsilon>0$, by $M \in \delta_{2}$, there exist $k>0$, $a>0, i_{0} \in \mathbb{N}$ and $\left\{c_{i}\right\}_{i=i_{0}+1}^{\infty}$ with $\sum_{i=i_{0}+1}^{\infty} c_{i}<\infty$ which satisfy

$$
M_{i}(u / \varepsilon) \leq k M_{i}(u)+c_{i} \quad\left(i>i_{0}, M_{i}(u) \leq a\right) .
$$

Since $\sum_{i=i_{0}+1}^{\infty} c_{i}<\infty$, there exists $i_{1} \in \mathbb{N}$ such that $\sum_{i=i_{1}+1}^{\infty} c_{i}<1 / 3$. By $x_{n}(i) \rightarrow$ $0\left(i=1,2, \ldots, i_{1}\right)$, there exists $n_{0} \in \mathbb{N}$ such that $\sum_{i=1}^{i_{1}} M_{i}\left(x_{n}(i) / \varepsilon\right)<1 / 3$ when $n \geq n_{0}$. Moreover, since $\rho_{M}\left(x_{n}\right) \rightarrow 0$, there exists $n_{1} \in \mathbb{N}$ such that $\rho_{M}\left(x_{n}\right)<\min \{1 / 3 k, a\}$ when 
$n \geq n_{1}$. Therefore, when $n \geq \max \left\{n_{0}, n_{1}\right\}$, we have

$$
\begin{aligned}
\sum_{i=1}^{\infty} M_{i}\left(\frac{x_{n}(i)}{\varepsilon}\right) & =\sum_{i=1}^{i_{1}} M_{i}\left(\frac{x_{n}(i)}{\varepsilon}\right)+\sum_{i=i_{1}+1}^{\infty} M_{i}\left(\frac{x_{n}(i)}{\varepsilon}\right) \\
& \leq \frac{1}{3}+\sum_{i=i_{1}+1}^{\infty}\left(k M_{i}\left(x_{n}(i)\right)+c_{i}\right) \\
& \leq \frac{1}{3}+k \cdot \frac{1}{3 k}+\frac{1}{3}=1 .
\end{aligned}
$$

It follows that $\left\|x_{n}\right\|<\varepsilon$, i.e. $\left\|x_{n}\right\| \rightarrow 0(n \rightarrow \infty)$.

LEMMA 7 ([1]). If $M \in \delta_{2}$, then $B(i)=\infty$.

\section{Results}

ThEOREM 1. A point $x \in S\left(l_{M}\right)$ is a strongly U-point if and only if

(1) $|x(i)|=B(i)(i \in \mathbb{N})$ or $\rho_{M}(x)=1$,

(2) $\xi(x)<1$

(3) (i) If for any $i \in \mathbb{N},|x(i)| \in S C_{M_{i}}$, then there do not exist $i, j \in \mathbb{N}$ with $i \neq j$ such that $|x(i)| \in S C_{M_{i}}^{+}$and $|x(j)| \in S C_{M_{j}}^{-}$,

(ii) If there exists $i_{0} \in \mathbb{N}$ such that $\left|x\left(i_{0}\right)\right| \notin S C_{M_{i_{0}}}$, then $|x(j)| \in S C_{M_{j}}^{0}$ for any $j \in \mathbb{N}$ with $j \neq i_{0}$,

(4) If $e(i)>0$, then $e(i)<|x(i)| \quad(i=1,2, \ldots)$.

Proof. Without loss of generality, we may assume that $x(i) \geq 0(i \in \mathbb{N})$.

We suppose (1) does not hold, then there exists $i_{0} \in \mathbb{N}$ such that $x\left(i_{0}\right)<B\left(i_{0}\right)$ and $\rho_{M}(x)<1$. Furthermore, we can find a real number $\lambda>0$ such that

$$
M_{i_{0}}\left(x\left(i_{0}\right)+\lambda\right) \leq 1-\sum_{i \neq i_{0}} M_{i}(x(i)) .
$$

Put

$$
y(i)=\left\{\begin{array}{ll}
x(i), & i \neq i_{0}, \\
x\left(i_{0}\right)+\lambda, & i=i_{0},
\end{array} \quad z(i)= \begin{cases}x(i), & i \neq i_{0}, \\
x\left(i_{0}\right)-\lambda, & i=i_{0} .\end{cases}\right.
$$

It is obvious that $y+z=2 x$ and $y \neq z$. But $\rho_{M}(y)=\sum_{i \neq i_{0}} M_{i}(x(i))+M_{i_{0}}\left(x\left(i_{0}\right)+\lambda\right) \leq 1$, hence $\|y\| \leq 1$. Similarly, we also have $\|z\| \leq 1$. Using $\|y+z\|=2$, we get $\|y\|=\|z\|=1$. This means that $x$ is not an extreme point. Since a strong U-point must be an extreme point, this is a contradiction.

Let us prove the necessity of condition (2). Otherwise, $\xi(x)=1$ i.e. $\rho_{M}(\lambda x)=\infty$ for any $\lambda>1$. Since $\|x\|=1$, there exists $i_{0} \in \mathbb{N}$ such that $x\left(i_{0}\right) \neq 0$. Put

$$
y(i)= \begin{cases}x(i), & i \neq i_{0}, \\ 0, & i=i_{0} .\end{cases}
$$

It is obvious that $\rho_{M}(\lambda y)=\infty$ for any $\lambda>1$, whence $\|y\| \geq 1$. On the other hand, clearly $\|y\| \leq\|x\|=1$. So we have $\|y\|=1$. Consequently, $1 \geq\left\|\frac{1}{2}(x+y)\right\| \geq\left\|\frac{1}{2}(y+y)\right\|=2$, hence $\|x+y\|=2$. But $x \neq y$, which contradicts that $x$ is a strong U-point. 
If the condition (i) of (3) does not hold, then there exist $i, j \in \mathcal{N}$ such that $x(i) \in$ $S C_{M_{i}}^{+}$and $x(j) \in S C_{M_{j}}^{-}$. For convenience we may assume $i=1, j=2$ and $x(1)=b_{1}$, $x(2)=a_{2}$ where $b_{1} \in S C_{M_{1}}^{+}, a_{2} \in S C_{M_{2}}^{-}$, then there exist $a_{1}>0$ and $b_{2}>0$ such that

$$
M_{1}(u)=A_{1} u+B_{1} \quad \text { for } u \in\left[a_{1}, b_{1}\right]
$$

and

$$
M_{2}(u)=A_{2} u+B_{2} \quad \text { for } u \in\left[a_{2}, b_{2}\right] .
$$

Take $\varepsilon_{1}>0$ and $\varepsilon_{2}>0$ such that $b_{1}-\varepsilon_{1} \in\left(a_{1}, b_{1}\right), a_{2}+\varepsilon_{2} \in\left(a_{2}, b_{2}\right)$ and $A_{1} \varepsilon_{1}=A_{2} \varepsilon_{2}$. Let

$$
y=\left(x(1)-\varepsilon_{1}, x(2)+\varepsilon_{2}, x(3), x(4), \ldots\right)
$$

Then

$$
\begin{aligned}
\rho_{M}(y) & =M_{1}\left(x(1)-\varepsilon_{1}\right)+M_{2}\left(x(2)+\varepsilon_{2}\right)+\sum_{i \geq 3} M_{i}(x(i)) \\
& =A_{1}\left(x(1)-\varepsilon_{1}\right)+B_{1}+A_{2}\left(x(2)+\varepsilon_{2}\right)+B_{2}+\sum_{i \geq 3} M_{i}(x(i)) \\
& =M_{1}(x(1))+M_{2}(x(2))+\sum_{i \geq 3} M_{i}(x(i))=\rho_{M}(x)=1 .
\end{aligned}
$$

So by the definition of the Luxemburg norm, we have $\|y\|=1$. Similarly,

$$
\begin{aligned}
\rho_{M}\left(\frac{x+y}{2}\right) & =M_{1}\left(x(1)-\frac{\varepsilon_{1}}{2}\right)+M_{2}\left(x(2)+\frac{\varepsilon_{2}}{2}\right)+\sum_{i \geq 3} M_{i}(x(i)) \\
& =M_{1}(x(1))+M_{2}(x(2))+\sum_{i \geq 3} M_{i}(x(i))=\rho_{M}(x)=1,
\end{aligned}
$$

i.e. $\left\|\frac{x+y}{2}\right\|=1$. Since $x \neq y, x$ is not a strong U-point. A contradiction.

We suppose the condition (ii) of (3) is not true. Then there exists $i_{0} \in \mathbb{N}$ such that $\left|x\left(i_{0}\right)\right| \notin S C_{M_{i_{0}}}$ and $j \in \mathbb{N}, j \neq i_{0}$ such that $x(j) \notin S C_{M_{j}}^{0}$. i.e. $x(j) \notin S C_{M_{j}}$ or $x(j) \in$ $S C_{M_{j}}^{+}$or $x(j) \in S C_{M_{j}}^{-}$. So, we can repeat the procedure from the proof of the necessity of the condition (i) of (3).

Let us finally prove the necessity of (4). Otherwise, there exists $i_{0} \in \mathbb{N}$ such that $e\left(i_{0}\right)>0$ and $x\left(i_{0}\right) \leq e\left(i_{0}\right)$. Let us consider two cases:

CASE I: $x\left(i_{0}\right)=e\left(i_{0}\right)$. Put

$$
y(i)= \begin{cases}x(i), & i \neq i_{0} \\ \frac{x\left(i_{0}\right)}{2}, & i=i_{0}\end{cases}
$$

Since $x\left(i_{0}\right)=e\left(i_{0}\right)<B\left(i_{0}\right)$, in virtue of $(1)$ we have $\rho_{M}(x)=1$. Therefore, we have the following equality

$$
\rho_{M}(y)=\sum_{i \neq i_{0}} M_{i}(x(i))+M_{i_{0}}\left(\frac{x\left(i_{0}\right)}{2}\right)=\sum_{i \neq i_{0}} M_{i}(x(i))+M_{i_{0}}\left(x\left(i_{0}\right)\right)=\rho_{M}(x)=1 .
$$

So $\|y\|=1$. Similarly,

$\rho_{M}\left(\frac{x+y}{2}\right)=\sum_{i \neq i_{0}} M_{i}(x(i))+M_{i_{0}}\left(\frac{3}{4} x\left(i_{0}\right)\right)=\sum_{i \neq i_{0}} M_{i}(x(i))+M_{i_{0}}\left(x\left(i_{0}\right)\right)=\rho_{M}(x)=1$,

i.e. $\|x+y\|=2$. But obviously $x \neq y$, which contradicts the fact that $x$ is a strong $\mathrm{U}$-point. 
CASE II: $x\left(i_{0}\right)<e\left(i_{0}\right)$. We put

$$
y(i)=\left\{\begin{array}{ll}
x(i), & i \neq i_{0}, \\
x\left(i_{0}\right)+\frac{e\left(i_{0}\right)-x\left(i_{0}\right)}{2}, & i=i_{0},
\end{array} \quad z(i)= \begin{cases}x(i), & i \neq i_{0}, \\
x\left(i_{0}\right)-\frac{e\left(i_{0}\right)-x\left(i_{0}\right)}{2}, & i=i_{0} .\end{cases}\right.
$$

It is obvious that $y+z=2 x$ and $y \neq z$. In the same way as in case I, it is easy to prove that $\|y\|=\|z\|=1$. Therefore, $x$ is not an extreme point, which leads to a contradiction.

Sufficiency. Let $x, y \in S\left(l_{M}\right)$ with $\|x+y\|=2$, we consider the following two cases:

CAse I: $|x(i)|=B(i)$ for all $i \in \mathbb{N}$. Without loss of generality, we may assume $x(i) \geq 0$ and $y(i) \geq 0(i=1,2, \ldots)$. In this case we have $\|(B(1), B(2), \ldots)\|=\|(x(1), x(2), \ldots)\|$ $=\|x\|=1$. Using

$$
x(i)+y(i) \leq 2 B(i) \quad(i=1,2, \ldots)
$$

and

$$
2=\|x+y\| \leq 2\|(B(1), B(2), \ldots)\|=2
$$

we have the equality $x(i)=B(i)(i=1,2, \ldots)$. Therefore $y(i)=x(i)=B(i)$ for all $i \in \mathbb{N}$ i.e. $x=y$.

CASE II: $\rho_{M}(x)=1$. First, we will prove that $\rho_{M}\left(\frac{x+y}{2}\right)=1$.

For any $\varepsilon \in\left(0, \frac{1-\xi(x)}{1+\xi(x)}\right)$ we have $\left\|(1+\varepsilon) \frac{x+y}{2}\right\|=1+\varepsilon$ and $\rho_{M}\left(\frac{1+\varepsilon}{1-\varepsilon} x\right)<\infty$. Hence there exists $\alpha>0$ such that

$$
\rho_{M}\left(\frac{1+\varepsilon}{1-\varepsilon} x\right)=\rho_{M}(x)+\alpha \varepsilon
$$

Therefore

$$
\begin{aligned}
1 & <\rho_{M}\left((1+\varepsilon) \frac{x+y}{2}\right)=\rho_{M}\left(\frac{1+\varepsilon}{2} y+\frac{1-\varepsilon}{2} \frac{1+\varepsilon}{1-\varepsilon} x\right) \\
& \leq \frac{1+\varepsilon}{2} \rho_{M}(y)+\frac{1-\varepsilon}{2} \rho_{M}\left(\frac{1+\varepsilon}{1-\varepsilon} x\right) \\
& =\frac{1+\varepsilon}{2} \rho_{M}(y)+\frac{1-\varepsilon}{2}\left(\rho_{M}(x)+\alpha \varepsilon\right) .
\end{aligned}
$$

Letting $\varepsilon \rightarrow 0$, we get $\rho_{M}(y)=1$. Since $\left\|\frac{x+y}{2}\right\|=1$ and the norm $\|\cdot\|_{M}$ is a convex function, it follows that $\|\cdot\|_{M}$ is an affine function on the segment between $x$ and $y$. Therefore

$$
\left\|\frac{\left(\frac{1}{2}(x+y)+x\right)}{2}\right\|=\left\|\frac{1}{4} y+\frac{3}{4} x\right\|=1 .
$$

Hence we can get in the same way as above (with $\frac{1}{2}(x+y)$ in place of $y$ ) that $\rho_{M}\left(\frac{x+y}{2}\right)=1$. Hence

$$
\begin{aligned}
0 & =\frac{\rho_{M}(x)+\rho_{M}(y)}{2}-\rho_{M}\left(\frac{x+y}{2}\right) \\
& =\sum_{i=1}^{\infty}\left[\left(\frac{M_{i}\left(x(i)+M_{I}(Y(i)\right.}{2}\right)-M_{i}\left(\frac{x(i)+y(i)}{2}\right)\right] \geq 0 .
\end{aligned}
$$


Thus we have

$$
\frac{M_{i}(x(i))+M_{i}(y(i))}{2}=M_{i}\left(\frac{x(i)+y(i)}{2}\right), \quad i=1,2,3, \ldots .
$$

This means that $x(i)=y(i)$ or $x(i)$ and $y(i)$ belong to the same intervals of $S A I\left(M_{i}\right)$ for all $i \in \mathbb{N}$.

If the condition (i) of (3) holds true, we may assume without loss of generality that $x, y \geq 0$ and either $x(i) \in S C_{M_{i}}^{+}$or $x(i) \in S C_{M_{i}}^{0}$ for all $i \in \mathbb{N}$. Define

$$
N_{1}=\left\{i \in \mathbb{N}: x(i) \in S C_{M_{i}}^{+}\right\} .
$$

In view of condition (4), we get, for any $i \in \mathbb{N}$, that there exist $A_{i}>0, B_{i} \in \mathbb{R}$ and $\varepsilon_{i}>0$ such that $M_{i}(u)=A_{i} u+B_{i}$ for all $u \in\left[x(i)-\varepsilon_{i}, x(i)\right]$. Therefore by the above properties of $x$ and $y$, we have

$$
\begin{array}{ll}
y(i)=x(i) & \left(\forall i \in \mathbb{N} \backslash N_{1}\right), \\
y(i) \leq x(i) & \left(\forall i \in N_{1}\right) .
\end{array}
$$

The equality $\rho_{M}\left(\frac{x+y}{2}\right)=\rho_{M}(x)$ implies that

$$
\sum_{i \in N_{1}} M_{i}\left(\frac{x(i)+y(i)}{2}\right)=\sum_{i \in N_{1}} M_{i}(x(i))
$$

i.e.

$$
\sum_{i \in N_{1}}\left(A_{i} \frac{x(i)+y(i)}{2}+B_{i}\right)=\sum_{i \in N_{1}}\left(A_{i} x(i)+B_{i}\right)
$$

Hence

$$
\sum_{i \in N_{1}} A_{i}\left(\frac{y(i)-x(i)}{2}\right)=0 .
$$

Consequently, $y(i)=x(i)$ for all $i \in \mathbb{N}$, i.e. $x=y$.

If (ii) of (3) holds, then $x(i)=y(i)$ for $i \neq i_{0}$. Moreover, by condition (4), there exist $A_{0}>0, B_{0} \in \mathbb{R}$ and $\varepsilon_{0}>0$ such that

$$
M_{i_{0}}(u)=A_{0} u+B_{0}, \quad u \in\left[x\left(i_{0}\right)-\varepsilon_{0}, x\left(i_{0}\right)+\varepsilon_{0}\right] .
$$

The equality $\rho_{M}\left(\frac{x+y}{2}\right)=\rho_{M}(x)$ implies $M_{i_{0}}\left(\frac{x\left(i_{0}\right)+y\left(i_{0}\right)}{2}\right)=M_{i_{0}}\left(x\left(i_{0}\right)\right)$, i.e.

$$
A_{0}\left(\frac{x\left(i_{0}\right)+y\left(i_{0}\right)}{2}\right)+B_{0}=A_{0}\left(x\left(i_{0}\right)\right)+B_{0} .
$$

Hence $x\left(i_{0}\right)=y\left(i_{0}\right)$ and so $x=y$. This finishes the proof of the theorem.

Theorem 2. If $x \in S\left(l_{M}\right)$, then the following statements are equivalent:

1. $x$ is a CLUR-point,

2. $x$ is a WCLUR-point,

3. (i) $M \in \delta_{2}$

(ii) $M \in \bar{\delta}_{2}$ or $\{i \in \mathbb{N}:|x(i)| \in(a, b]\}=\emptyset$ where $[a, b] \in S A I\left(M_{i}\right)$.

Proof. The implication $1 \Rightarrow 2$ is obvious. 
$2 \Rightarrow 3$. We suppose (i) does not hold, i.e. $M \notin \delta_{2}$. By Lemma 2 , there exist $z \in l_{M}$ and a singular function $\Phi$ with $\rho_{M}(z)<\infty$ and $\Phi(x-z) \neq 0$. Set

$$
x_{n}=(x(1), \ldots, x(n), z(n+1), z(n+2), \ldots) \quad(n=1,2, \ldots) .
$$

Then

$$
\rho_{M}\left(x_{n}\right) \leq \rho_{M}(x)+\sum_{i=n+1}^{\infty} M_{i}(z(i)) \rightarrow \rho_{M}(x) \leq 1,
$$

so $\lim \sup _{n \rightarrow \infty}\left\|x_{n}\right\| \leq 1$. Notice $\left\|x_{n}+x\right\| \geq 2\|(x(1), \ldots, x(n), 0, \ldots)\| \rightarrow 2$, we have $\liminf _{n \rightarrow \infty}\left\|x_{n}+x\right\| \geq 2$. Hence $\left\|x_{n}\right\| \rightarrow 1$ and $\left\|x_{n}+x\right\| \rightarrow 2(n \rightarrow \infty)$. Since $x_{n} \rightarrow x$ coordinatewise, we may assume without loss of generality that $x_{n} \stackrel{w}{\rightarrow} x$ (passing to a subsequence if necessary). But $\Phi\left(x-x_{n}\right)=\Phi(x-z) \neq 0$, which contradicts $x_{n} \stackrel{w}{\rightarrow} x$. This contradiction shows that $M \in \delta_{2}$.

Without loss of generality, we assume $x(i) \geq 0$ for all $i \in \mathbb{N}$.

If the condition (ii) of (3) does not hold, then there exists $j \in \mathbb{N}$ such that $x(j) \in(a, b]$, without loss of generality we may assume $j=1$ and $M \notin \bar{\delta}_{2}$ where $[a, b] \in S A I\left(M_{1}\right)$ satisfies $M_{1}(u)=A u+B$ for $u \in[a, b]$. Take $\varepsilon>0$, such that $x(1)-\varepsilon \in(a, b]$. Since $M \notin \bar{\delta}_{2}$, by Lemma 3 , there exist $u_{i}^{n}>0$ satisfying

$$
M_{i}\left(u_{i}^{n}\right) \leq \frac{1}{n}, \quad M_{i}\left(\frac{u_{i}^{n}}{2}\right)>\left(1-\frac{1}{n}\right) \frac{M_{i}\left(u_{i}^{n}\right)}{2} \quad\left(i=m_{n-1}+1, \ldots, m_{n}\right)
$$

and

$$
\sum_{i=m_{n-1}+1}^{m_{n}} M_{i}\left(u_{i}^{n}\right)>1 .
$$

Without loss of generality, we may assume $A \varepsilon<1$. For every sufficiently large $n$, take $m_{n-1}<m_{n}^{\prime} \leq m_{n}$ such that

$$
A \varepsilon-\frac{1}{2^{n}} \leq \sum_{i=m_{n-1}+1}^{m_{n}^{\prime}} M_{i}\left(u_{i}^{n}\right)<A \varepsilon, \quad n=1,2, \ldots
$$

Let $\left\{e_{n}\right\}_{n}$ be the natural basis of $l^{1}$ and $\left\{p_{n}\right\}_{n}$ the projections $p_{n}(x)=\sum_{i=1}^{n} x(i) e_{i}$ for $x=(x(i))_{i} \in l_{M}$. Put

$$
x_{n}=P_{n} x-P_{1} x+(x(1)-\varepsilon) e_{1}+\sum_{i=m_{n-1}+1}^{m_{n}^{\prime}} u_{i}^{n} e_{i}
$$

Then

$$
\begin{aligned}
\rho_{M}\left(x_{n}\right) & =M_{1}(x(1)-\varepsilon)+\sum_{i=2}^{n} M_{i}(x(i))+\sum_{i=m_{n-1}+1}^{m_{n}^{\prime}} M_{i}\left(u_{i}^{n}\right) \\
& =\alpha x(1)-\alpha \varepsilon+\beta+\sum_{i=2}^{n} M_{i}(x(i))+\sum_{i=m_{n-1}+1}^{m_{n}^{\prime}} M_{i}\left(u_{i}^{n}\right)
\end{aligned}
$$




$$
\begin{aligned}
& =M_{1}(x(1))-\alpha \varepsilon+\sum_{i=2}^{n} M_{i}(x(i))+\sum_{i=m_{n-1}+1}^{m_{n}^{\prime}} M_{i}\left(u_{i}^{n}\right) \\
& <\sum_{i=1}^{n} M_{i}(x(i))-A \varepsilon+A \varepsilon=\sum_{i=1}^{n} M_{i}(x(i)) \leq 1 .
\end{aligned}
$$

So $\lim \sup _{n \rightarrow \infty}\left\|x_{n}\right\| \leq 1$. Moreover,

$$
\begin{aligned}
\rho_{M}\left(\frac{x+x_{n}}{2}\right) & \geq M_{1}\left(x(1)-\frac{\varepsilon}{2}\right)+\sum_{i=2}^{n} M_{i}(x(i))+\sum_{i=m_{n-1}+1}^{m_{n}^{\prime}} M_{i}\left(\frac{x(i)+u_{i}^{n}}{2}\right) \\
& \geq \sum_{i=1}^{n} M_{i}(x(i))-\frac{A \varepsilon}{2}+\sum_{i=m_{n-1}+1}^{m_{n}^{\prime}}\left(\left(1-\frac{1}{n}\right) \frac{M_{i}\left(u_{i}^{n}\right)}{2}\right) \\
& \geq \sum_{i=1}^{n} M_{i}(x(i))-\frac{A \varepsilon}{2}+\frac{1}{2}\left(1-\frac{1}{n}\right)\left(A \varepsilon-\frac{1}{2^{n}}\right) \rightarrow 1(n \rightarrow \infty) .
\end{aligned}
$$

Hence $\liminf _{n \rightarrow \infty}\left\|\frac{x+x_{n}}{2}\right\| \geq 1$. Thus we have $\left\|x_{n}\right\| \rightarrow 1$ and $\left\|x_{n}+x\right\| \rightarrow 2(n \rightarrow \infty)$.

Since $\lim _{n \rightarrow \infty}\left(A \varepsilon-1 / 2^{n}\right)=A \varepsilon>A \varepsilon / 2$, there exists $n_{0}$ such that $A \varepsilon-1 / 2^{n}>A \varepsilon / 2$ when $n \geq n_{0}$. Therefore

$$
\left\|x_{m}-x_{n}\right\| \geq\left\|\sum_{i=m_{m-1}+1}^{m_{m}^{\prime}} u_{i}^{m} e_{i}\right\| \geq \sum_{i=m_{m-1}+1}^{m_{m}^{\prime}} M_{i}\left(u_{i}^{m}\right)>A \varepsilon-\frac{1}{2^{m}}>\frac{A \varepsilon}{2}
$$

when $m>n \geq n_{0}$.

This means that $\left\{x_{n}\right\}$ is not compact in $S\left(l_{M}\right)$, hence $x$ is not a CLUR-point. But, by $M \in \delta_{2}$ and Theorem 2 in [7], we can get that $l_{M}$ has H-property. Therefore $x$ is not a WCLUR-point. This is a contradiction.

$3 \Rightarrow 1$. Suppose $x \in S\left(l_{M}\right),\left\{x_{n}\right\}_{n=1}^{\infty} \subset S\left(l_{M}\right)$ and $\left\|x_{n}+x\right\| \rightarrow 2(n \rightarrow \infty)$. In order to complete this proof we distinguish two cases.

(I) $M \in \delta_{2} \cap \bar{\delta}_{2}$. In this case, by Lemma 1 and Lemma 2, we take $\left\{f_{n}\right\} \subset S\left(l_{N}^{0}\right)$ such that $f_{n}\left(x_{n}+x\right)=\left\|x_{n}+x\right\| \rightarrow 2(n \rightarrow \infty)$. Then

$$
f_{n}(x) \rightarrow 1 \quad \text { and } \quad f_{n}\left(x_{n}\right) \rightarrow 1 \quad(n \rightarrow \infty)
$$

In virtue of [6], $l_{N}^{0}$ is reflexive. Then there is a subsequence $\left\{f_{n_{i}}\right\}$ of $\left\{f_{n}\right\}$ and $f \in l_{N}^{0}$ such that $f_{n} \rightarrow^{w} f$. It is obvious that in virtue of $\lim _{n \rightarrow \infty} f_{n}(x)=1$ this yields $f(x)=1$. Hence $\|f\|^{0}=1$. By Theorem 1 in [7], we get that $l_{N}^{0}$ has H-property. Hence $\left\|f_{n}-f\right\|^{0}$ $\rightarrow 0(n \rightarrow \infty)$. So

$$
f\left(x_{n_{i}}\right)=\left(f-f_{n_{i}}\right)\left(x_{n_{i}}\right)+f_{n_{i}}\left(x_{n_{i}}\right) \rightarrow 1 \quad(n \rightarrow \infty) .
$$

Using now the reflexivity of $l_{M}$, we can find a subsequence $\left\{x_{n_{i}}^{\prime}\right\} \subset\left\{x_{n_{i}}\right\}$ and $x^{\prime} \in l_{M}$ such that $x_{n_{i}}^{\prime} \rightarrow^{w} x^{\prime} \quad(n \rightarrow \infty)$. Obviously $f\left(x^{\prime}\right)=1$, whence $\left\|x^{\prime}\right\|=1$. By the property $\mathrm{H}$ for $l_{M}$, we have $\lim _{n \rightarrow \infty}\left\|x_{n_{i}}^{\prime}-x^{\prime}\right\|=0$, i.e. $\left\{x_{n}\right\}$ is compact in $S\left(l_{M}\right)$, which implies that $x$ is a CLUR-point. 
(II) $M \in \delta_{2}$ and $\{i \in \mathbb{N}:|x(i)| \in(a, b]\}=\emptyset \quad$ where $[a, b] \in S A I\left(M_{i}\right)$. First, we will prove that $x_{n}(i) \rightarrow x(i)$ for all $i \in \mathbb{N}$. We first show

$$
\liminf _{n} x_{n}(j) \geq x(j), \quad j=1,2, \ldots
$$

If not, there exist $j_{0} \in \mathbb{N}, \varepsilon_{0}>0$ and a subsequence of $\left\{x_{n}\right\}$, denoted again by $\left\{x_{n}\right\}$, such that

$$
x_{n}\left(j_{0}\right) \leq x\left(j_{0}\right)-\varepsilon .
$$

Since $x\left(j_{0}\right) \notin(a, b]$, there exists $\delta>0$ such that

$$
M_{j_{0}}\left(\frac{x\left(j_{0}\right)+x_{n}\left(j_{0}\right)}{2}\right) \leq(1-\delta) \frac{M_{j_{0}}\left(x\left(j_{0}\right)\right)+M_{j_{0}}\left(x_{n}\left(j_{0}\right)\right)}{2} .
$$

Then by Lemma 4 and Lemma 5, we get

$$
\begin{aligned}
0 & \leftarrow \frac{\rho_{M}(x)+\rho_{M}\left(x_{n}\right)}{2}-\rho_{M}\left(\frac{x_{n}+x}{2}\right) \\
& =\sum_{i=1}^{\infty}\left[\frac{M_{i}\left(x_{n}(i)\right)+M_{i}(x(i))}{2}-M_{i}\left(\frac{x_{n}(i)+x(i)}{2}\right)\right] \\
& \geq \frac{M_{j_{0}}\left(x_{n}\left(j_{0}\right)\right)+M_{j_{0}}\left(x\left(j_{0}\right)\right)}{2}-M_{j_{0}}\left(\frac{x_{n}\left(j_{0}\right)+x\left(j_{0}\right)}{2}\right) \\
& \geq \delta \frac{M_{j_{0}}\left(x_{n}\left(j_{0}\right)\right)+M_{j_{0}}\left(x\left(j_{0}\right)\right)}{2} \geq \frac{\delta}{2} M\left(\frac{\varepsilon}{2}\right)>0 .
\end{aligned}
$$

This contradiction shows that condition (1) holds.

Now, we will show that

$$
\limsup _{n} x_{n}(j) \leq x(j), \quad j=1,2, \ldots
$$

Otherwise, there exist $j_{0} \in \mathbb{N}$ and $\varepsilon>0$ such that $\lim \sup _{n} x_{n}\left(j_{0}\right) \geq x\left(j_{0}\right)+\varepsilon$. Then $\lim \sup _{n} M_{j_{0}}\left(x_{n}\left(j_{0}\right)\right) \geq M_{j_{0}}\left(x\left(j_{0}\right)\right)+\varepsilon^{\prime}$ for some $\varepsilon^{\prime}>0$. Hence

$$
\begin{aligned}
1 & =\limsup _{n} \rho_{M}\left(x_{n}\right)=\underset{n}{\limsup } \sum_{i \neq j_{0}} M_{i}\left(x_{n}(i)\right)+M_{j_{0}}\left(x_{n}\left(j_{0}\right)\right) \\
& \geq \sum_{i \neq j_{0}} M_{i}(x(i))+M_{j_{0}}\left(x\left(j_{0}\right)\right)+\varepsilon^{\prime}=\rho_{M}(x)+\varepsilon^{\prime}=1+\varepsilon^{\prime} .
\end{aligned}
$$

This is a contradiction. So $\lim _{n \rightarrow \infty} x_{n}(i)=x(i)(i \in \mathbb{N})$ thanks to (1) and (2).

Next, we will show that $\rho_{M}\left(\frac{x_{n}-x}{2}\right) \rightarrow 0(n \rightarrow \infty)$. In fact, for any $\varepsilon>0$, there exist $i_{0}$ and $n_{0}$ such that

$$
\sum_{i>i_{0}} M_{i}(x(i))<\frac{\varepsilon}{4}, \quad \sum_{i=1}^{i_{0}} M_{i}\left(\frac{x_{n}(i)-x(i)}{2}\right)<\frac{\varepsilon}{4}
$$

and

$$
\sum_{i=1}^{i_{0}} \mid M_{i}\left(x_{n}(i)-M_{i}(x(i)) \mid<\varepsilon \quad \text { when } n \geq n_{0} .\right.
$$


Hence when $n \geq n_{0}$,

Therefore

$$
\begin{aligned}
\sum_{i>i_{0}} M_{i}\left(x_{n}(i)\right) & =\rho_{M}\left(x_{n}\right)-\sum_{i=1}^{i_{0}} M_{i}\left(x_{n}(i) \leq 1-\sum_{i=1}^{i_{0}} M_{i}(x(i))+\varepsilon\right. \\
& \leq 1-\left(1-\sum_{i>i_{0}} M_{i}(x(i))\right)+\varepsilon<\frac{5}{4} \varepsilon .
\end{aligned}
$$

$$
\begin{aligned}
\rho_{M}\left(\frac{x_{n}-x}{2}\right) & =\sum_{i=1}^{i_{0}} M_{i}\left(\frac{x_{n}(i)-x(i)}{2}\right)+\sum_{i>i_{0}} M_{i}\left(\frac{x_{n}(i)-x(i)}{2}\right) \\
& \leq \sum_{i=1}^{i_{0}} M_{i}\left(\frac{x_{n}(i)-x(i)}{2}\right)+\frac{1}{2}\left[\sum_{i>i_{0}} M_{i}\left(x_{n}(i)\right)+\sum_{i>i_{0}} M_{i}(x(i))\right] \\
& <\frac{\varepsilon}{4}+\frac{1}{2}\left(\frac{5}{4} \varepsilon+\frac{\varepsilon}{4}\right)=\varepsilon,
\end{aligned}
$$

i.e. $\rho_{M}\left(\frac{x_{n}-x}{2}\right) \rightarrow 0(n \rightarrow \infty)$. So by $\lim _{n \rightarrow \infty} x_{n}(i)=x(i)(i \in \mathbb{N})$ and Lemma 6 , we get $\left\|x_{n}-x\right\| \rightarrow 0(n \rightarrow \infty)$. This means $x$ is a CLUR-point. Thus, the proof is finished.

It is obvious that a point $x \in S(X)$ is a LUR-point if and only if it is a CLUR-point and a SU-point. So, in view of Lemma 7, Theorem 1 and Theorem 2, we easily obtain the following criteria for LUR-point of $S\left(l_{M}\right)$.

THEOREM 3. A point $x \in S\left(l_{M}\right)$ is a LUR-point if and only if:

1. $M \in \delta_{2}$,

2. If for any $i \in \mathbb{N},|x(i)| \in S C_{M_{i}}$, then

(i) $\left\{i \in \mathbb{N}:|x(i)| \in S C_{M_{i}}^{+}\right\}=\emptyset$;

(ii) if $\left\{i \in \mathbb{N}:|x(i)| \in S C_{M_{i}}^{+}\right\} \neq \emptyset$, then $\left\{\forall i \in \mathbb{N}:|x(i)| \in S C_{M_{i}}^{-}\right\}=\emptyset$ and $M \in \bar{\delta}_{2}$.

3. If there exists $i_{0} \in \mathbb{N}$ such that $\left|x\left(i_{0}\right)\right| \notin S C_{M_{i_{0}}}$, then $|x(j)| \in S C_{M_{j}}^{0}(j \in \mathbb{N}$, $\left.j \neq i_{0}\right)$ and $M \in \bar{\delta}_{2}$,

4. If $e(i)>0$, then $e(i)<|x(i)|$ for all $i \in \mathbb{N}$.

\section{References}

[1] S. Chen, Geometry of Orlicz spaces, Dissertationes Math. 356 (1996).

[2] J. Diestel, Geometry of Banach Spaces, Lecture Notes in Math. 485, Springer, 1975.

[3] J. Musielak, Orlicz Spaces and Modular Spaces, Lecture Notes in Math. 1034, Springer, 1983.

[4] J. Wang and T. Wang, WM property of Musielak-Orlicz sequence space, Fareast J. Math. Sci. 5 (1997), 475-496.

[5] H. Hudzik and Y. Ye, Support functionals and smoothness in Musielak-Orlicz spaces endowed with the Luxemburg norm, Comment. Math. Univ. Carolin. 31 (1990), 661-684.

[6] M. Denker and H. Hudzik, Uniform non-l(1) Musielak-Orlicz sequence spaces, Proc. Indian Acad. Sci. 101 (1991), 71-86.

[7] Y. Cui, Some convexities of Musielak-Orlicz sequence spaces, J. Math. (PRC) 15 (1995), 291-296. 